\title{
A network of reverberating neuronal populations encodes motor decision in macaque premotor cortex Maurizio Mattia*1,2, Pierpaolo Pani ${ }^{3}$, Giovanni Mirabella ${ }^{3}$, Stefania Costa ${ }^{2}$, Paolo Del Giudice ${ }^{1}$ and Stefano Ferraina ${ }^{3}$
}

\author{
Address: ${ }^{1}$ Department of Technologies and Health, Istitituto Superiore di Sanità, Rome, Italy, ${ }^{2}$ Dottorato di Ricerca in Neurofisiologia, Sapienza \\ University, Rome, Italy and ${ }^{3}$ Department of Physiology and Pharmacology, Sapienza University, Rome, Italy \\ Email: Maurizio Mattia* - mattia@iss.infn.it \\ * Corresponding author
}

from Eighteenth Annual Computational Neuroscience Meeting: CNS*2009

Berlin, Germany. 18-23 July 2009

Published: 13 July 2009

BMC Neuroscience 2009, I0(Suppl I):OI4 doi:I0.II86/I47|-2202-I0-SI-OI4

This abstract is available from: http://www.biomedcentral.com/I47I-2202/I0/SI/OI4

(C) 2009 Mattia et al; licensee BioMed Central Ltd.

\section{Background}

We investigate, through in-vivo experiments and theoretical models, the dynamic mechanisms subserving motor decision tasks. The proposed computational framework is based on local reverberations in multiple neuronal subpopulations. Signatures of such reverberations are identified in the simulated multi-modular network of spiking neurons and recognized in the analysis of recorded neural activity. The reported experimental results are compatible with a neuronal substrate in which several local populations undergo sudden transitions as a late reaction to a visual stimulus. Often, such transitions are very plausibly supported by strong local recurrent feedback as in models of decision making [1]. Transitions also occur without clear evidence of local reverberation; the combined evidence suggests the coexistence of "active" and "passive" modules, the latter responding to the activity of the first ones, which together cooperate in order to represent a distributed and well stereotyped motor program.

\section{Results}

We recorded from dorsal premotor cortex (PMd) of two monkeys required to reach quickly a target randomly appearing in one of two opposite peripheral positions (movement conditions) after a go-signal (no-stop trials), but to withhold the movement whenever an intervening stop-signal was shown after a random delay (stop trials). We selected recordings showing a significant increase, in at least one movement condition, of MUA activity during no-stop reaction time (the epoch between the go signal and the movement onset). Sixty-one percent (68/112) of these recordings are characterized by a sharp upward transition (SUT) of the MUA signal, the time of which is strongly correlated with the movement onset at the single trial level. On average, upward transitions precede the movement onset by $110 \mathrm{~ms}$ and are completed in less than $100 \mathrm{~ms}$.

The predictive value of the SUT time of occurrence is strengthened by the behavioral outcome of the stop trials. In "wrong" stop trials, when the monkeys fail to cancel the movement, an early SUT is observed while either none or later SUT are observed in "correct" stop trials. Taken together the evidences from stop and no-stop trials support the speculation that the occurrence of a SUT in PMd is causally related to movement onset. In a sizable fraction of the recordings during the reaction times, the MUAs have a bimodal distribution, which suggests the existence of two preferred levels of firing rates; this is consistent with observations from single unit recordings [2] and compatible with attractor-like dynamics of the probed neuronal population $[1,3,4]$. We furthermore probed during reaction time the Fourier spectral features of the raw electrophysiological signal in $20 \mathrm{~ms}$ sliding windows. Low-activity and high-activity periods show clearly different modulations of the spectral power in medium (50- 
$230 \mathrm{HZ}$ ) and high-frequency (230-1080 Hz) bands. MUA with unimodal distributions do not show the above modulation of power spectra; their transitions can be thought to be driven by the input they receive from other reverberating modules.

Simulations of multiple populations of synaptically coupled leaky integrate-and-fire neurons with recurrent excitation and inhibition [4] reproduced qualitatively well the observed MUA dynamics. In particular, randomness in SUTs emerges from intrinsic fluctuation of spiking activity. For strong enough AMPA/NMDA recurrent couplings, simulations reproduced both bimodal distributions of firing rate and spectral modulations observed from experiments, reinforcing the picture of neural populations capable of multi-stable dynamics and high input susceptibility, supported by local activity reverberation, as a plausible interpretation of experimental evidence.

\section{References}

I. Wang $X$ J: Decision making in recurrent neuronal circuits. Neuron 2008, 60:215-234.

2. Churchland MM, Yu BM, Ryu SI, Santhanam G, Shenoy KV: Neural variability in premotor cortex provides a signature of motor preparation. J Neurosci 2006, 26:3697-37I2.

3. Zipser D, Kehoe B, Littlewort G, Fuster J: A spiking network model of short-term active memory. J Neurosci 1993, I 3:3406-3420.

4. Amit DJ, Brunel N: Dynamics of a recurrent network of spiking neurons before and following learning. Network 1997, 8:373-404.
Publish with Bio Med Central and every scientist can read your work free of charge

"BioMed Central will be the most significant development for disseminating the results of biomedical research in our lifetime. "

Sir Paul Nurse, Cancer Research UK

Your research papers will be:

- available free of charge to the entire biomedical community

- peer reviewed and published immediately upon acceptance

- cited in PubMed and archived on PubMed Central

- yours - you keep the copyright 